\title{
ON KUMMER'S TWENTY-FOUR SOLUTIONS OF THE HYPERGEOMETRIC DIFFERENTIAL EQUATION
}

BY

B. DWORK

\begin{abstract}
The $p$-adic analyticity of the Boyarsky matrix associated with the hypergeometric function $F(a, b ; c ; x)$ has been investigated in an earlier article. The transformation of this matrix under translation of $(a, b, c)$ by $\mathbf{Z}^{3}$ was determined at that time. This article gives the transformation of this matrix under the extended Kummer group. The $p$-adic implications of quadratic and higher-degree transformations remain open.
\end{abstract}

The object of this article is to explain the relation between Kummer's twenty-four solutions of this equation

$$
\left(\lambda(1-\lambda) \frac{d^{2}}{d \lambda^{2}}+(c-(a+b+1) \lambda) \frac{d}{d \lambda}-a b\right) y=0
$$

and the $p$-adic theory of this equation.

For simplicity of exposition let us assume that $a, b, c$ satisfy the condition

$$
a, b, c-a, c-b \text { all lie outside } \mathbf{Z} \text {. }
$$

The unique solution of (1) analytic at $\lambda=0$ is given by the classical formula

$$
F(a, b, c ; \lambda)=\sum_{s=0}^{\infty} \frac{(a)_{s}(b)_{s}}{(c)_{s} s !} \lambda^{s}
$$

where

$$
(a)_{s}=\Gamma(a+s) / \Gamma(a), \quad s \in \mathbf{N}, s \geqslant 0 .
$$

The Euler integral formula

$$
\begin{aligned}
& F(a, b, c ; \lambda)=\frac{\Gamma(c)}{\Gamma(b) \Gamma(c-b)} \int_{0}^{1} \omega_{a, b, c}, \\
& \omega_{a, b, c}=x^{b-1}(1-x)^{c-b-1}(1-x \lambda)^{-a} d x,
\end{aligned}
$$

valid for $\operatorname{Re} c>\operatorname{Re} b>0$ (a restriction which may be removed [7, p. 60, equation (13)] by changing the path of integration) shows that we are concerned with the periods of a certain differential. As is well known $[5,6$; 11, p. 107; 12] the Kummer list may be obtained by transformation of this integral representation. Indeed there

Received by the editors September 1, 1982 and, in revised form, September 28, 1983.

1980 Mathematics Subject Classification. Primary 12B40, 34A30.

(C)1984 American Mathematical Society $0002-9947 / 84 \$ 1.00+\$ .25$ per page 
are twenty-four fractional linear transformations which map the set $\{0,1, \infty\}$ into the set $\{0,1, \infty, 1 / \lambda\}$. Under such a substitution, $x=(\alpha t+\beta) /(\gamma t+\delta)$, the differential $\omega_{a, b, c}(x, \lambda)$ is transformed into a differential which (up to a factor independent of $\lambda, t)$ is of the form

$$
\lambda^{\nu_{0}}(1-\lambda)^{\nu_{1}} \omega_{\hat{\hat{a}}, \hat{b}, \hat{\hat{c}}}(t, \hat{\lambda}),
$$

where $\bmod \mathbf{Z}, \hat{\hat{a}}, \hat{\hat{b}}, \hat{\hat{c}}$ are linear functions of $(a, b, c)$. We refer to the group of mappings $(a, b, c) \mapsto(\hat{\hat{a}}, \hat{b}, \hat{\hat{c}}) \bmod \mathbf{Z}$ as the Kummer group. It is group theoretically isomorphic to the octahedral group. The mappings $\lambda \rightarrow \hat{\lambda}$ form a noncommutative group of order 6 well known as the group of fractional linear transformations which permute the points $\{0,1, \infty\}$.

In this way, (cf. Table I) one deduces a solution

$$
\lambda^{\nu_{0}}(1-\lambda)^{\nu_{1}} F(\hat{\hat{a}}, \hat{\hat{b}}, \hat{\hat{c}}, \hat{\lambda})
$$

of (1) with constant factor adjusted so that we have six sets of solutions, each set consisting of four solutions which coincide at $\hat{\lambda}=0$ and represent an eigenvector of the local monodromy at $\hat{\lambda}=0$.

As noted by Riemann and re-examined by the more recent authors previously mentioned, the element $F(b, a, c ; x)$ cannot be obtained in this way, i.e., the mapping $(a, b, c) \mapsto(b, a, c)$ does not lie in the Kummer group. By adjoining this element we obtain a group of 48 elements which we call the extended Kummer group. Since many authors (Kummer, Ince, Bateman project, Whittaker and Watson, Goursat, Poole) do not distinguish between these two groups and since the standard references do not give consistent lists from this point of view, we provide such a list in Table I as an introduction to the calculations summarized in Table II.

We have made available [3] a very lengthy treatment of the action of Frobenius upon the solutions of (1) subject to the further hypothesis

$$
(a, b, c) \in \mathbf{Q} \cap \mathbf{Z}_{p} .
$$

In particular the associated $2 \times 2$ matrix is (aside from possible poles at $0, \infty$ ) analytic in the region

$$
|\lambda-1|>|\pi|=|p|^{1 / p-1} .
$$

More recently [4], we constructed the associated Boyarsky matrix $\Gamma_{B}(a, b, c, \lambda)$ which we showed to be analytic as a function of $(a, b, c)$ in each of the $p^{3}$ polydisks

$$
\left\{(a, b, c)\left|(a, b, c)+\left(\mu_{1}, \mu_{2}, \mu_{3}\right)\right|<|p|^{1-e_{2}}\right\},
$$

where

$$
e_{2}=\frac{1}{(p-1)}\left(1-\frac{1}{p}-\frac{1}{p-1}\right), \quad \mu_{i} \in \mathbf{N}, \quad 0 \leqslant \mu_{i}<p \quad(i=1,2,3) .
$$

This analyticity of $\Gamma_{B}$ was demonstrated subject to the condition

$$
|\lambda|=1=|\lambda-1| \text {. }
$$

We showed that the contiguity relations of Gauss correspond to the transformation laws of $\Gamma_{B}$ under translation of $(a, b, c)$ by elements of $\mathbf{Z}^{3}$. In the present work 
we show that Kummer's list correspond to the transformation laws of $\Gamma_{B}(a, b, c ; \lambda)$ under the twenty-four mappings $(a, b, c) \mapsto(\hat{a}, \hat{b}, \hat{c})$ listed in Table I. One of the applications is the analyticity of $\Gamma_{B}$ as a function of $(a, b, c, \lambda)$ with $\lambda$ not restricted by (10). Another application (not developed here) is the possibility of eliminating the determinant calculation [4, equation (3.22.8)] from the proof [4, Theorem 3.22] of analyticity of $\Gamma_{B}$. Finally, we use duality $[3, \S 4.7]$ to determine the relation between $\Gamma_{B}(a, b, c, \lambda)$ and $\Gamma_{B}(b, a, c, \lambda)$. Putting

$$
(a, b, c)=-\left(\mu_{1}, \mu_{2}, \mu_{3}\right)+p\left(a^{\prime}, b^{\prime}, c^{\prime}\right),
$$

$\left(a^{\prime}, b^{\prime}, c^{\prime}\right)$ bounded by $|p|^{-e_{2}}$, we find

$$
\zeta(a, b, c)\left(\begin{array}{cc}
c-a, & 0 \\
0, & c-b
\end{array}\right) \Gamma_{B}(a, b, c ; \lambda)=\Gamma_{B}(b, a, c ; \lambda)\left(\begin{array}{cc}
c^{\prime}-a^{\prime}, & 0 \\
0, & c^{\prime}-b
\end{array}\right),
$$

where $\zeta(a, b, c)$ is a scalar function of $(a, b, c)$ independent of $\lambda$. Thus by a determinant calculation at $\lambda=0, \zeta$ may be determined up to \pm 1 . A precise determination is given in $\S 6$.

In this way we determine the transformation laws of $\Gamma_{B}$ under the group extension of $\mathbf{Z}^{3}$ deduced from the 48 transformations generated by Table $I$ and the transformation $(a, b, c) \mapsto(b, a, c)$.

We acknowledge the influence upon this article of the work [1] of Adolphson and Sperber on Bessel functions. We observe that subsection 3.2 is an ad hoc treatment in a special case of results known to Monsky and Washnitzer.

Notation. $\Omega$ is an algebraically closed extension of $\mathbf{Q}_{p}$ complete under a valuation extending that of $\mathbf{Q}_{p}$,

$$
\begin{aligned}
& \pi^{p^{-1}}=p \text {. } \\
& D_{1}(a, r)=\{x \in \Omega|| x-a \mid<r\} \text {. } \\
& f_{\vec{a}}(x, \lambda)=x^{a_{2}-1}(1-x)^{a_{3}-a_{2}}(1-x \lambda)^{-a_{1}} \text {. } \\
& \omega_{\vec{a}, \lambda}=f_{\vec{a}}(x, \lambda) d x /(1-x) \text {. } \\
& X_{\vec{a}}(x, \lambda)=f_{\vec{a}}(x, \lambda)\left(\begin{array}{c}
1 \\
(1-x)^{-1}
\end{array}\right) d x \text {. } \\
& \underline{L}_{\lambda}=\Omega[x, 1 / x, 1 /(1-x), 1 /(1-x \lambda)] \text {. }
\end{aligned}
$$

$$
|x|>\varepsilon_{0}, \quad|1-x|>\varepsilon, \quad|1-\lambda x|>\varepsilon_{1 / \lambda}, \quad|1 / x|>\varepsilon_{\infty} .
$$

$\operatorname{ord}\left(a_{1}, a_{2}, a_{3}\right)=\operatorname{Inf}_{i}$ ord $a_{i}$.

ord $x=-\log |x| / \log p$.

The * of an invertible matrix denotes the transpose of the inverse.

$\gamma_{p}(a, b)$ is defined for $a, b \in \mathbf{Q} \cap \mathbf{Z}_{p}-\mathbf{Z}, p b-a \in \mathbf{Z}$ to be $\Gamma_{p}(a) \pi^{\mu}(-\pi)^{b-b_{0}}$, where $\mu \in\{0,1, \ldots, p-1\} \mu \equiv p b-a \bmod p, p b_{0}-a=\mu$ (cf. [3, equation (21.4.2); 4, equation (1.6)]).

$$
e_{2}=\left(1-p^{-1}-(p-1)^{-1}\right)(p-1)^{-1} \text {. }
$$

1. Resumé of earlier work. We assume initially that

$$
\begin{aligned}
& \vec{a}=\left(a_{1}, a_{2}, a_{3}\right) \in\left(\mathbf{Q} \cap \mathbf{Z}_{p}\right)^{3}, \\
& a_{1}, a_{2}, a_{3}-a_{1}, a_{3}-a_{2} \text { all outside } \mathbf{Z} .
\end{aligned}
$$


The vector space $\omega_{\vec{a}} \underline{L}_{\lambda} / d\left(f_{\vec{a}} \underline{L}_{\lambda}\right)$ is of dimension two with $\omega_{\vec{a}}$ and $(1-x) \omega_{\vec{a}}$ as a basis. We write

$$
X_{\vec{a}}(x, \lambda)=\left(\begin{array}{c}
(1-x) \omega_{\vec{a}} \\
\omega_{\vec{a}}
\end{array}\right)
$$

If then $\vec{c} \equiv \vec{a} \bmod \mathbf{Z}$, we may consider the change of basis matrix $B$ defined by

$$
\left(\begin{array}{c}
(1-x) \omega_{\vec{c}} \\
\omega_{\vec{c}}
\end{array}\right)=X_{\vec{c}}(x, \lambda)=B(\vec{a}, \vec{c}, \lambda) X_{\vec{a}}(x, \lambda) .
$$

We have shown that $B$ depends rationally upon $\vec{a}$ and $\lambda$; explicit formulae are given elsewhere $[4, \S 3]$.

For

$$
\begin{gathered}
\lambda \neq 0, \infty, \quad|\lambda-1|>|\pi|, \\
p \vec{b}-\vec{a}=\vec{\mu} \in \mathbf{Z}^{3}, \\
\vec{b} \in\left(Q \cap \mathbf{Z}_{p}\right)^{3}
\end{gathered}
$$

we have defined an analytic lifting $\psi_{1}$ of the Cartier mapping which maps $\omega_{\vec{a}, \lambda} L_{\lambda}$ into $\omega_{\vec{b}, \lambda^{p}} L_{\lambda^{p}}$ by setting for $\xi \in L_{\lambda}$,

$$
\psi_{1}\left(\xi x f_{\alpha}(x, \lambda) \frac{d x}{x}\right)=\psi_{0}\left(\frac{\xi f_{\vec{a}}(x, \lambda)}{f_{\vec{b}}\left(x^{p}, \lambda^{p}\right)}\right) x f_{\vec{b}}\left(x, \lambda^{p}\right) \frac{d x}{x},
$$

where we identify $F_{\vec{a}, \vec{b}}(x, \lambda)=f_{\vec{a}}(x, \lambda) / f_{\vec{b}}\left(x^{p}, \lambda^{p}\right)$ with that element of $L_{\lambda}$ which represents the indicated algebraic function of $x$ and which, for a "generic" value of $x$, is approximated by

$$
x^{p-1-\mu_{2}}(1-x)^{\mu_{2}-\mu_{3}}(1-\lambda x)^{\mu_{1}}
$$

and where $\psi_{0}$ is the mapping of $L_{\lambda}$ into $L_{\lambda^{p}}$ defined by

$$
\left(\psi_{0} \xi\right)(x)=\sum_{y^{p}=x} \xi(y) / p y^{p-1} .
$$

By passage to quotients we deduce from $\psi_{1}$ a mapping of $\omega_{\vec{a}, \lambda} L_{\lambda} / d\left(f_{\vec{a}}(x, \lambda) L_{\lambda}\right)$ onto $\omega_{\vec{b}, \lambda^{p}} L_{\lambda^{p}} / d\left(f_{\vec{b}}\left(x, \lambda^{p}\right) L_{\lambda^{p}}\right)$ whose matrix $\gamma(\vec{a}, \vec{b} ; \lambda)$ we specify by writing

$$
\psi_{1} X_{\vec{a}}(x, \lambda)=\gamma(\vec{a}, \vec{b} ; \lambda) X_{\vec{b}}\left(x, \lambda^{p}\right) \bmod d\left(f_{\vec{b}}\left(x, \lambda^{p}\right) L_{\lambda^{p}}\right) .
$$

With $\vec{a}, \vec{b}$ satisfying (1.1), (1.4) we have shown [3]:

1.7. The matrix $\gamma(\vec{a}, \vec{b}, \lambda)$ is an analytic function of $\lambda$ on the set (1.3), with at most poles at $\lambda=0, \infty$.

The precise nature of this function at $\lambda=0$ has been determined in [3, Chapters 24, 25].

We define $\Gamma_{B}(\vec{a}, \lambda)$ for $\vec{a}$ satisfying (1.1) and $\lambda$ satisfying (1.3) by writing

$$
\Gamma_{B}(\vec{a}, \lambda)=\gamma(\vec{a}, \vec{b}, \lambda),
$$

where we now insist that (1.4) holds with $\vec{\mu}=\left(\mu_{1}, \mu_{2}, \mu_{3}\right)$,

$$
0 \leqslant \mu_{i} \leqslant p-1, \quad i=1,2,3 .
$$

We state the main result of our previous investigation [4, Theorem 3.22]. 
1.9. THEOREM. For

$$
|\lambda|=1=|\lambda-1|
$$

$\Gamma_{B}$ extends to an analytic function of $\vec{a}$ for each fixed $\vec{\mu}$ satisfying (1.8.1) on the set

$$
\text { ord } b_{i}>-e_{2} \quad(i=1,2,3) .
$$

1.10. Indeed, using the fact that the elements of

$$
\underline{0}=\mathbf{Z}_{p}\left[\frac{1}{1-\lambda}, \frac{1}{1-\lambda^{p}}, \frac{1}{p}\left(\frac{1}{(\lambda-1)^{p}}-\frac{1}{\lambda^{p}-1}\right)\right]
$$

are bounded by 1 for $|\lambda-1| \geqslant 1$, the formulae used in the proof of 1.9 show that $\Gamma_{B}$ extends to a function analytic in the pair $(\vec{b}, \lambda)$ for $\vec{b}$ restricted by (1.9.2) and $\lambda$ restricted to the set

$$
\{\lambda|| \lambda-1 \mid \geqslant 1, \lambda \neq \infty\}
$$

with singularities at $\lambda=\infty$ being at worst pole of order $\mu_{1}$.

1.11. For $\vec{a} \in \Omega^{3}$ we define

$$
G_{\vec{a}}(\lambda)=\left(\begin{array}{cc}
-\frac{a_{3}}{\lambda} & \frac{a_{3}-a_{1}}{1-\lambda} \\
\frac{a_{3}-a_{2}}{\lambda} & \frac{a_{1}+a_{2}-a_{3}}{1-\lambda}
\end{array}\right) .
$$

We recall the equation

$$
\frac{d}{d \lambda}\left(u_{1}, u_{2}\right)=\left(u_{1}, u_{2}\right) G_{\vec{a}}(\lambda)
$$

is satisfied by

(1.11.3) $\left(u_{1}, u_{2}\right)=\left(\left(a_{3}-a_{2}\right) F\left(a_{1}, a_{2}, a_{3}+1 ; \lambda\right), a_{3} F\left(a_{1}, a_{2}, a_{3} ; \lambda\right)\right)$.

For $z \neq 0,1, \infty$, we define $C_{\vec{a}}(z, \lambda)$ to be the solution matrix of $(1.11 .2)_{\vec{a}}$ which is normalized by the condition

$$
C_{\vec{a}}(z, z)=I .
$$

Thus $C_{\vec{a}}(z, \lambda)$ is an analytic function of $\lambda$ for $(z-\lambda)$ sufficiently small. For $\vec{a}$ satisfying (1.1), this matrix converges for

$$
|\lambda-z|<|z| \operatorname{Min}(1,|z-1|) \text {. }
$$

We observe that if $\lambda_{0}, \lambda$ both satisfy (1.11.5) then

$$
C_{\vec{a}}(z, \lambda)=C_{\vec{a}}\left(z, \lambda_{0}\right) C_{\vec{a}}\left(\lambda_{0}, \lambda\right)
$$

since both sides satisfy (1.11.2) $)_{\vec{a}}$ and coincide at $\lambda=\lambda_{0}$. Putting $z=\lambda$ we conclude that

$$
C_{\vec{a}}\left(\lambda_{0}, \lambda\right)=C_{\vec{a}}\left(\lambda, \lambda_{0}\right)^{-1} \text {. }
$$

1.12. The deformation theory $[3$, Chapter $4, \S 6]$ may be interpreted as stating that if $\vec{a}, \vec{z}, \vec{\lambda}$ satisfy (1.1), (1.11.5), then

$$
X_{\vec{a}}(x, \lambda) \equiv C_{\vec{a}}(z, \lambda)^{t} X_{\vec{a}}(x, z) \quad \bmod d\left(f_{\vec{a}}(x, z) L_{z}\right) .
$$


This is equivalent to the statement

$$
\frac{\partial}{\partial \lambda} X_{\vec{a}}(x, \lambda) \equiv G_{\vec{a}}(\lambda)^{t} X_{\vec{a}}(x, \lambda) \bmod d\left(f_{\vec{a}}(x, \lambda) \underline{L}_{\lambda}\right)
$$

The relation between (1.11.2) and the matrix $\gamma$ (equation (1.6)) may be written

$$
C_{\vec{b}}\left(z^{p}, \lambda^{p}\right) \gamma(\vec{a}, \vec{b}, \lambda)^{t}=\gamma(\vec{a}, \vec{b}, z)^{t} C_{\vec{a}}(z, \lambda) .
$$

This may be deduced from (1.12.1) and (1.6).

1.13 Since one of our main tools is the deformation matrix $C_{\vec{a}}(z, \lambda)$ of subsection 1.11 , we discuss the convergence without assuming validity of (1.1).

Lemma. The matrix $C_{\vec{a}}(z, \lambda)$ is analytic as a function of $\vec{a}, z, \lambda$ subject to the condition

$$
\operatorname{Inf}(0, \operatorname{ord} \vec{a})-\operatorname{Sup}(0, \operatorname{ord} z, \operatorname{ord}(1-z))+\operatorname{ord}(\lambda-z)>\frac{1}{p-1} .
$$

Proof. By a formal calculation, starting with (1.11.2), we find

$$
\frac{d^{s}\left(u_{1}, u_{2}\right)}{d \lambda^{s}}=\left(u_{1}, u_{2}\right) G_{\vec{a}}^{(s)}(\lambda)
$$

where the coefficients of $G_{\vec{a}}^{(s)}$ are polynomials over $\mathbf{Z}$ in $\vec{a}, 1 / \lambda, 1 /(1-\lambda)$ of degree bounded by $s$ in each variable. By Taylor's theorem, for $z \neq 0,1, \infty$

$$
C_{\vec{a}}(z, \lambda)=\sum_{s=0}^{\infty} \frac{1}{s !} G_{a}^{(s)}(z) \cdot(\lambda-z)^{s} .
$$

The assertion follows immediately.

1.14. If $x, \lambda, t$ are related (say rationally) then let $(\partial / \partial \lambda) x(\operatorname{resp} .(\partial / \partial \lambda) t)$ designate differentiation with respect to keeping $\lambda$ (resp. $t$ ) fixed. An elementary calculation shows that for any locally analytic function $g$ of $(x, \lambda)$ we have

$$
\left(\frac{\partial}{\partial \lambda}\right)_{t}(g d x)=\left(\frac{\partial}{\partial \lambda}\right)_{x}(g d x)+d\left(g \cdot\left(\frac{\partial x}{\partial \lambda}\right)_{t}\right) .
$$

Thus for cohomological purposes we need not distinguish between the two possible definitions of $\partial / \partial \lambda$.

1.15. Corrections of [4].

In equation (3.14), $\hat{A}_{s, t}$ should read $\hat{A}_{s, \tau}$.

On p. 140 , line $1^{*}, T_{1}$ should read $T_{1 / \lambda}$.

In equation (3.15.1), $\lambda^{1+\mu_{2}-p}$ should read $\lambda^{1+\mu_{2}}$.

2. The Kummer transformations. In Table I we list 24 fractional linear transformations, $T_{j}$, which map the set $\{0,1, \infty\}$ into the set $\{0,1, \infty, 1 / \lambda\}$. Clearly there will be one point, $x_{0}$, not in the image. We define $1 / \theta_{m}(\lambda)$ to be $T_{m}^{-1}\left(x_{0}\right)$, the inverse image under $T_{m}$ of the missing point. The mappings $\left\{\theta_{m}\right\}$ form the six fractional linear transformations which permute the points $\{0 ; 1, \infty\}$. The twenty-four transformations $\left\{T_{m}\right\}$ do not form a group. The six elements $T_{1}, T_{3}, T_{5}, T_{7}, T_{9}, T_{11}$ do form a group, $\mathscr{T}$, and the full set consists of translates $T_{j} \mathscr{T}, j=1,2,6,10$, the classification being according to the four possible choices of excluded element $x_{0}$.

In this section we will assume that $\vec{a}, \vec{b}, \lambda$ satisfy (1.1), (1.4) and (1.9.1). 
Now let

$$
x=T_{m}(t, \lambda), \quad 1 \leqslant m \leqslant 24 .
$$

We could write subject to (1.1)

$$
X_{\vec{a}}(x, \lambda) \equiv H_{m}(\vec{a}, \lambda) X_{M_{m}(\vec{a})}\left(t, \theta_{m}(\lambda)\right) \bmod d\left(f_{M_{m}(\vec{a})}\left(t, \theta_{m}(\lambda)\right) \underline{L}_{\theta_{m}(\lambda)}(t)\right)
$$

with $M_{m}(\vec{a})$ unique modulo $\mathbf{Z}^{3}$ and $H_{m}$ a $2 \times 2$ matrix independent of $x$ and depending algebraically upon $\lambda$, with precise value depending upon the actual choice of $M_{m}(\vec{a})$. However, calculations show that we may choose $M_{m}(\vec{a})$ such that $(2.2)$ may be replaced by an equality (valid incidentally for all $\vec{a}$ )

$$
X_{\vec{a}}(x, \lambda)=H_{m}(\vec{a}, \lambda) X_{M_{m}(\vec{a})}\left(t, \theta_{m}(\lambda)\right),
$$

and this construction fixes $M_{m}(\vec{a})=\left(\hat{a}_{1}, \hat{a}_{2}, \hat{a}_{3}\right)$ uniquely. Table II displays the determination. It is useful to observe that the matrix $H_{m}(\vec{a}, \lambda)$ may itself be described as a product

$$
H_{m}(\vec{a}, \lambda)=h_{m}(a, \lambda) N_{m}(\lambda)
$$

of a scalar function, $h_{m}$ depending upon $\vec{a}$ and $\lambda$, with a $2 \times 2$ matrix function $N_{m}$ depending rationally upon $\lambda$ and independent of $\vec{a}$. The factors cannot be described uniquely by these conditions, and a choice is given in Table II for each $m$. We write for each $m$, following a formal calculation,

$$
h_{m}(a, \lambda)=(-1)^{k(\vec{a})} \lambda^{k_{0}(\vec{a})}(1-\lambda)^{k_{1}(\vec{a})},
$$

where $k, k_{0}, k_{1}$ are linear (but not necessarily homogeneous) in $\vec{a}$ and so subject to (1.1) we are dealing with an algebraic function of $\lambda$. We make no attempt to specify a branch of this function, but subject to (1.1) and (1.4), (letting $\sigma$ denote the absolute Frobenius automorphism on the maximal unramified extension of $\mathbf{Q}_{p}$ ) we interpret the ratio

$$
h_{m}(\vec{a}, \vec{b}, \lambda) \stackrel{\operatorname{def}}{=} \frac{h_{m}(\vec{a}, \lambda)}{h_{m}^{\sigma}\left(\vec{b}, \lambda^{p}\right)}=(-1)^{k(\vec{a})-p k(\vec{b})} \lambda^{k_{0}(\vec{a})-p k_{0}(\vec{b})} \frac{(1-\lambda)^{k_{1}(\vec{a})}}{\left(1-\lambda^{p}\right)^{k_{1}(\vec{b})}}
$$

which is well defined for $\lambda$ not too close to 1 by the condition that

$$
(1-\lambda)^{k_{1}(\vec{a})} /\left(1-\lambda^{p}\right)^{k_{1}(\vec{b})}
$$

be approximated by $(1-\lambda)^{k_{1}(\vec{a})-p k_{1}(\vec{b})}$. A table of the linear functions $k, k_{0}, k_{1}$ may be deduced from Table II by observing the choice of $h_{m}$. In our application we shall be concerned with $h_{m}(\vec{a}, \vec{b}, \lambda)$ rather than with $h_{m}(\vec{a}, \lambda)$, and for this reason we have indicated $k(\vec{a})$ only $\bmod \mathbf{Z}$.

By differentiating (2.3) with respect to $\lambda$ (cf. 1.14)) and using equation (1.12.2) and letting $\lambda_{m}=\theta_{m}(\lambda)$ we find

$$
G_{\vec{a}}(\lambda)^{t} H_{m}(\vec{a}, \lambda)=\frac{d}{d \lambda} H_{m}(\vec{a}, \lambda)+\frac{d \theta_{m}}{d \lambda} H_{m}(\vec{a}, \lambda)\left(G_{M_{m}(\vec{a})}\left(\lambda_{m}\right)\right)^{t} .
$$




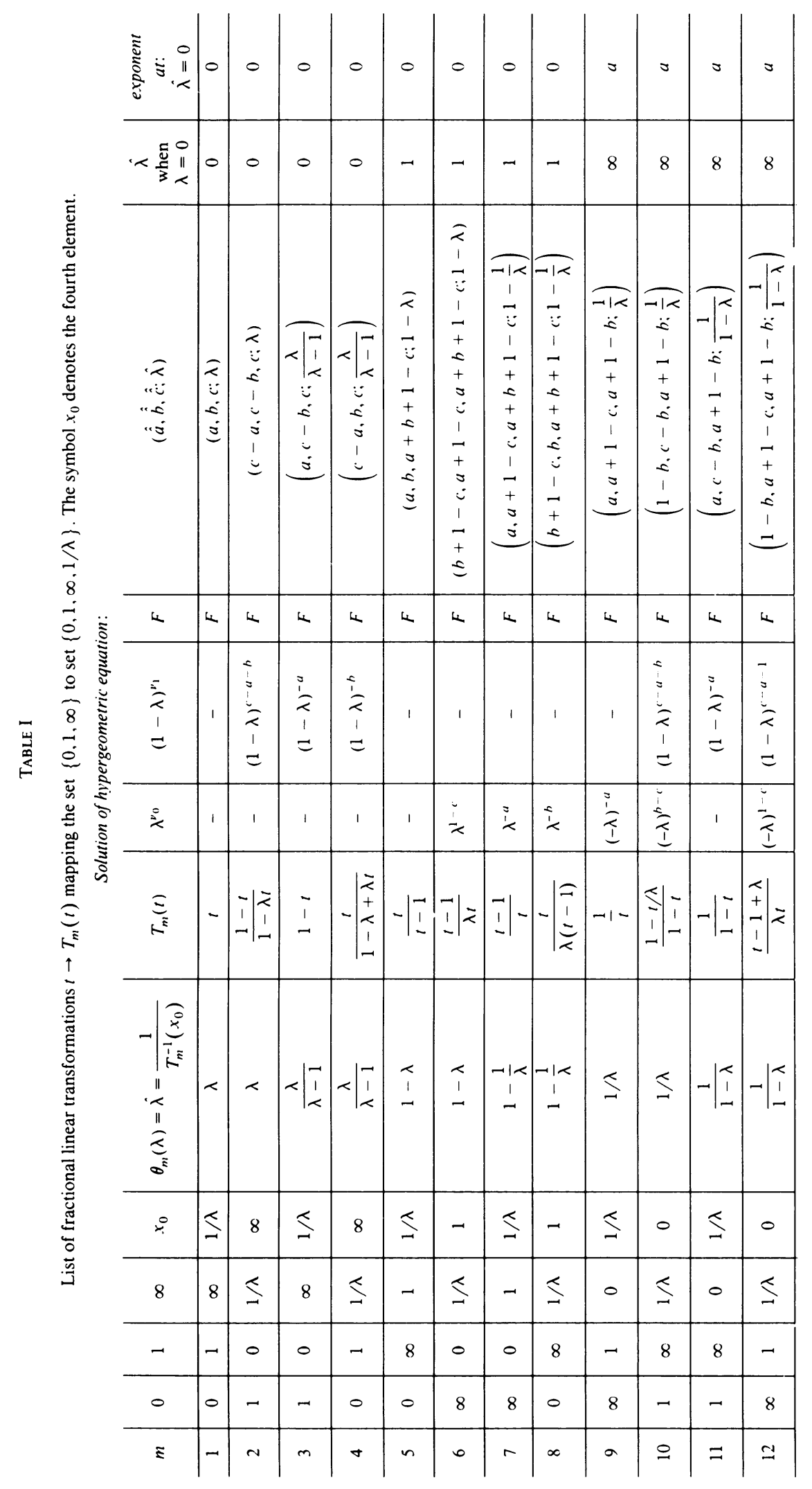




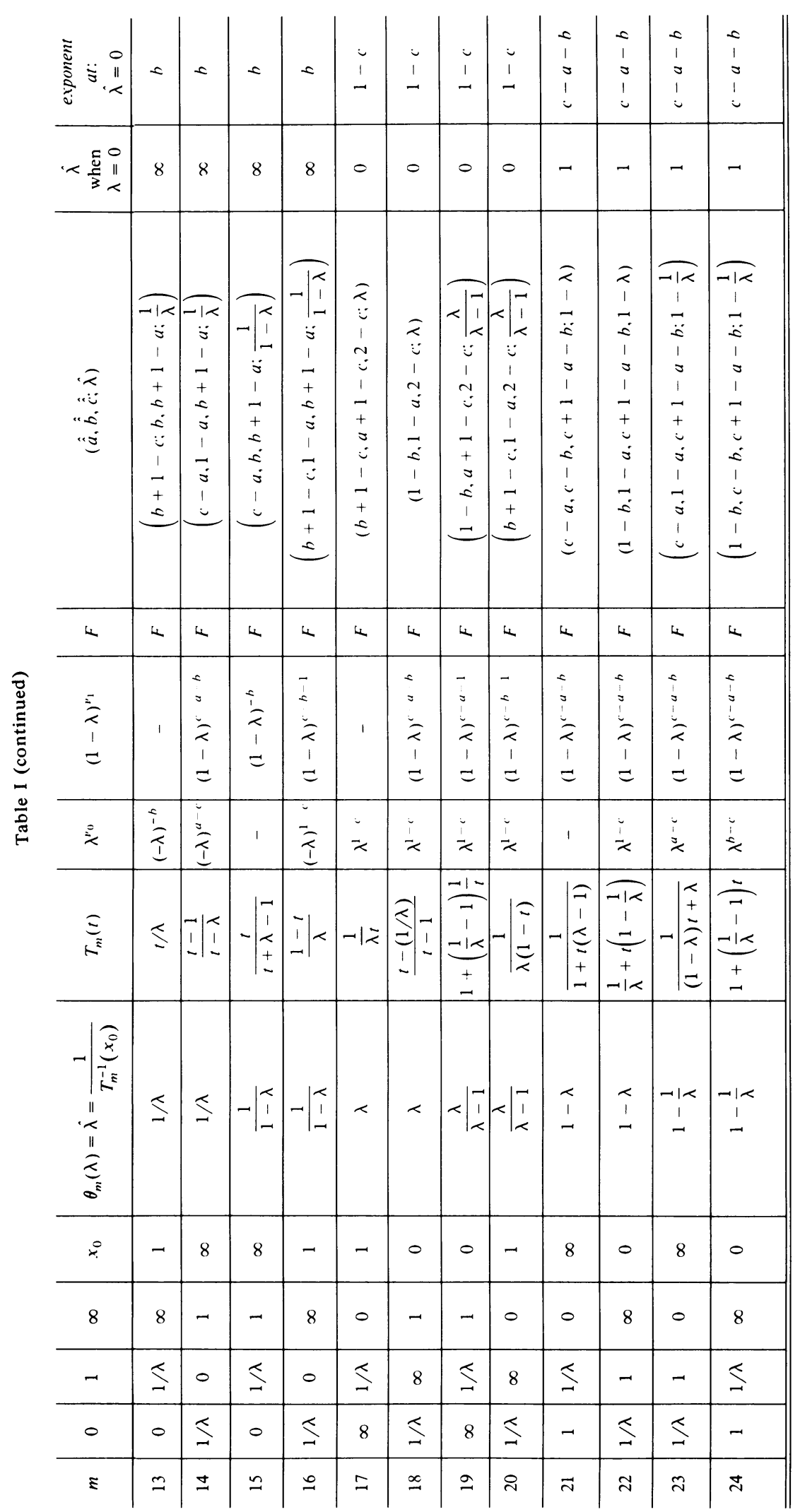




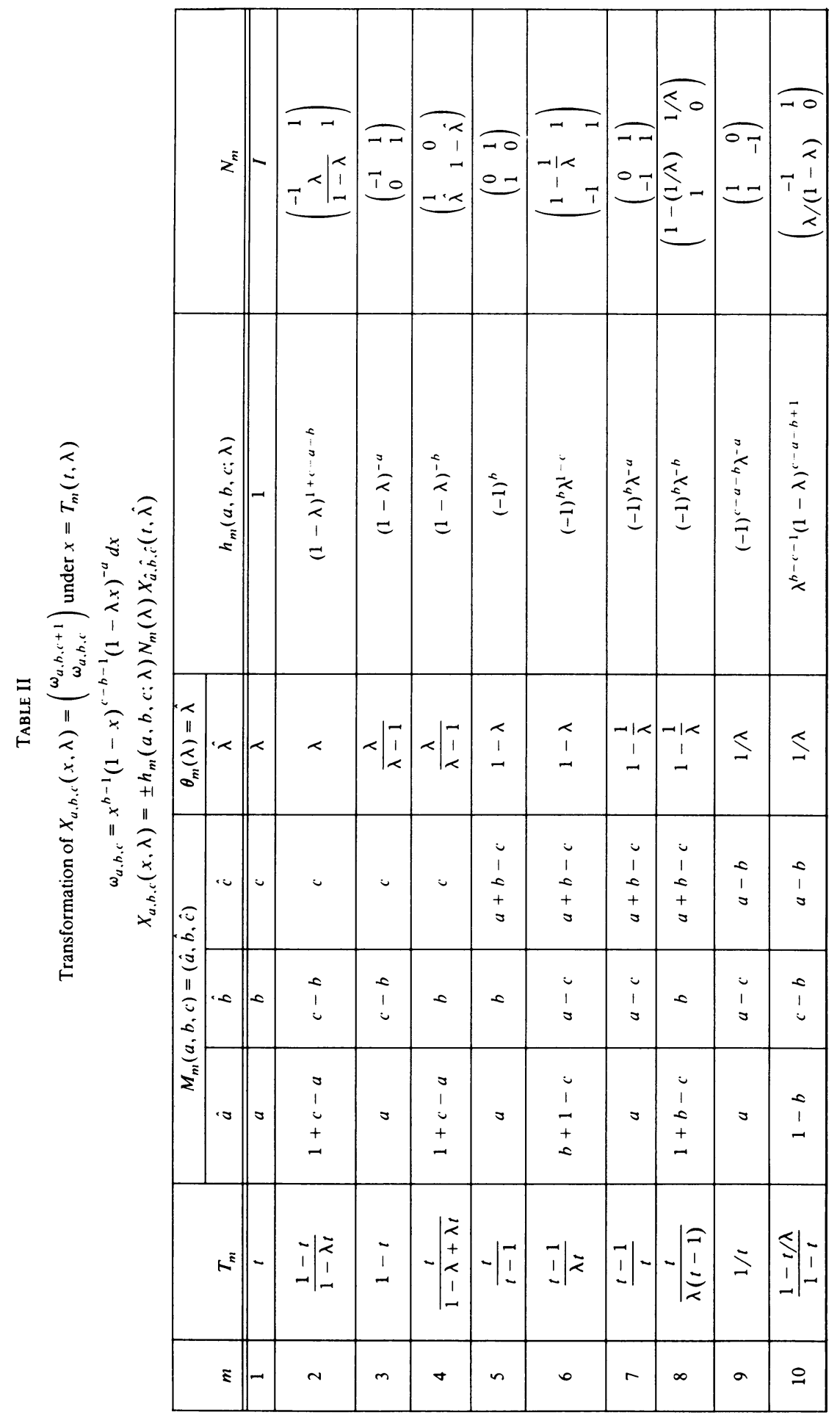




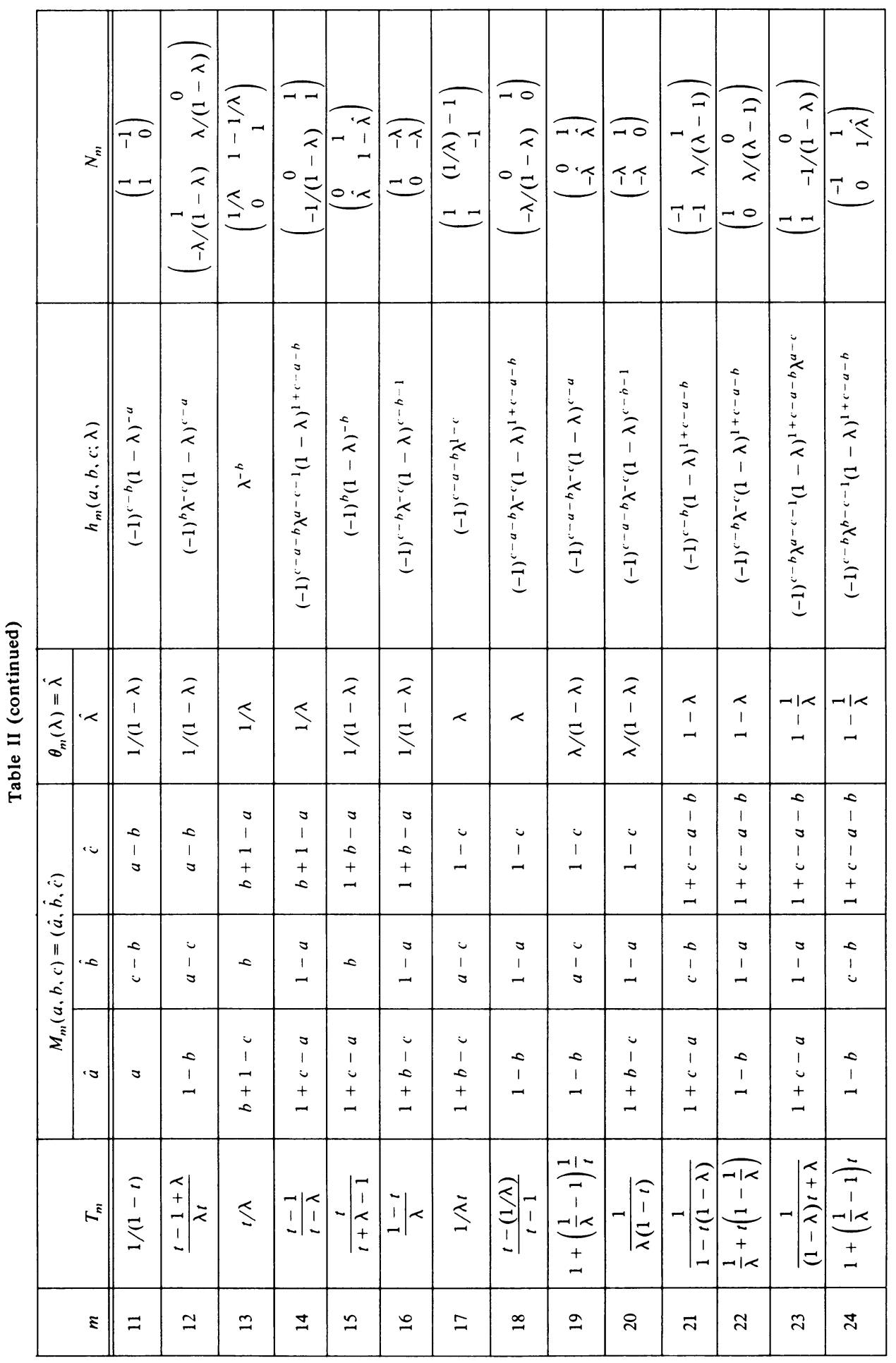


This shows that the mapping

$$
\left(v_{1}, v_{2}\right) \mapsto\left(u_{1}, u_{2}\right)=\left(v_{1}, v_{2}\right) H_{m}(\vec{a}, \lambda)^{t}
$$

maps solutions of the equation

$$
\frac{d}{d \lambda_{m}}\left(v_{1}, v_{2}\right)=\left(v_{1}, v_{2}\right) G_{M_{m}(\vec{a})}\left(\lambda_{m}\right)
$$

into solutions of (1.11.2) $)_{\vec{a}}$. In particular for $z \neq 0,1, \infty$

$$
H_{j}(\vec{a}, z)^{t} C_{\vec{a}}(z, \lambda)=C_{M_{j}(\vec{a})}\left(\theta_{j}(z), \theta_{j}(\lambda)\right) H_{j}(\vec{a}, \lambda)^{t},
$$

the equation being interpreted by means of the fact that for $\lambda$ close to $z$, the branch of the algebraic function $H_{j}$ is specified by its determination at $z$.

This being said, the validity of (2.9) follows from the fact that each side represents a solution matrix of $(1.11 .2)_{\vec{a}}$ and the sides coincide at $\lambda=z$.

We now define

$$
\begin{aligned}
K_{m}(\vec{a}, \vec{b}, \lambda)= & \gamma(\vec{a}, \vec{b}, \lambda)^{*} N_{m}\left(\lambda^{p}\right)^{*} C_{M_{m}(\vec{b})}\left(\theta_{m}\left(\lambda^{p}\right), \theta_{m}(\lambda)^{p}\right) \\
& \cdot \gamma\left(M_{m}(\vec{a}), M_{m}(\vec{b}), \theta_{m}(\lambda)\right)^{t} N_{m}(\lambda)^{t} h_{m}(\vec{a}, \vec{b}, \lambda),
\end{aligned}
$$

well defined for $\vec{a}, \vec{b}, \lambda$ satisfying (1.1), (1.4), (1.9.1). It may be continued analytically by means of Theorem 1.9 and subsection 1.13 so as to be well defined subject to (1.4.1), (1.9.1) provided

$$
\text { ord } \vec{b}>\operatorname{Sup}\left(-1+\frac{1}{p-1},-e_{2}\right) \text {. }
$$

2.11. THEOREM. $K_{m}(\vec{a}, \vec{b}, \lambda)$ is a scalar function of $(\vec{a}, \vec{b})$ independent of $\lambda$ provided $\mu_{3}$ does not lie between $\mu_{1}$ and $\mu_{2}$.

2.11.1. Note. In $\S 3$ we shall give a second analysis of $K_{m}$ which removes all restrictions on $\vec{\mu}$.

Proof. Let $z, \lambda$ satisfy (1.9.1), $|\lambda-z|$ sufficiently small. Let $a, b$ satisfy conditions (1.1) and (1.4). Then by (1.12.3)

$$
\begin{aligned}
& C_{M_{m}(\vec{b})}\left(\theta_{m}(z)^{p}, \theta_{m}(\lambda)^{p}\right) \gamma\left(M_{m}(\vec{a}), M_{m}(\vec{b}), \theta_{m}(\lambda)\right)^{t} \\
& \quad=\gamma\left(M_{m}(\vec{a}), M_{m}(\vec{b}), \theta_{m}(z)\right)^{t} C_{M_{m}(\vec{a})}\left(\theta_{m}(z), \theta_{m}(\lambda)\right) .
\end{aligned}
$$

By the transitivity property (1.11.6) we have

$$
\begin{aligned}
C_{M_{m}(\vec{b})}\left(\theta_{m}(z)^{p}, \theta_{m}(\lambda)^{p}\right)= & C_{M_{m}(\vec{b})}\left(\theta_{m}(z)^{p}, \theta_{m}\left(z^{p}\right)\right) C_{M_{m}(\vec{b})}\left(\theta_{m}\left(z^{p}\right), \theta_{m}\left(\lambda^{p}\right)\right) \\
& \cdot C_{M_{m}(\vec{b})}\left(\theta_{m}\left(\lambda^{p}\right), \theta_{m}(\lambda)^{p}\right)
\end{aligned}
$$

By (2.9) we have

$$
C_{M_{m}(\vec{b})}\left(\theta_{m}\left(z^{p}\right), \theta_{m}\left(\lambda^{p}\right)\right)=H_{m}\left(\vec{b}, z^{p}\right)^{t} C_{\vec{b}}\left(z^{p}, \lambda^{p}\right) H_{m}\left(\vec{b}, \lambda^{p}\right)^{*}
$$


Substituting this expression in the right side of (2.11.3) and substituting the resulting formula in the left side of (2.11.2) and replacing $C_{M_{m}(\vec{a})}\left(\theta_{m}(z), \theta_{m}(\lambda)\right)$ in the right side of (2.11.2) by the expression involving $C_{\vec{a}}(z, \lambda)$ deduced from (2.9), we obtain a lengthy equality which after multiplying on the left by

$$
\left(C_{M_{m}(\vec{b})}\left(\theta_{m}(z)^{p}, \theta_{m}\left(z^{p}\right)\right) H_{m}\left(\vec{b}, z^{p}\right)^{t}\right)^{-1}
$$

and on the right by $H_{m}(\vec{a}, \lambda)^{t}$ assumes the form

$$
C_{\vec{b}}\left(z^{p}, \lambda^{p}\right) \gamma(\vec{a}, \vec{b}, \lambda)^{t} K_{m}(\vec{a}, \vec{b}, \lambda)=\gamma(\vec{a}, \vec{b}, z)^{t} K_{m}(\vec{a}, \vec{b}, z) C_{\vec{a}}(z, \lambda) .
$$

Multiplying each side of this equation on the left by the inverse of the corresponding side of (1.12.3) we deduce

$$
K_{m}(\vec{a}, \vec{b}, \lambda)=C_{\vec{a}}(z, \lambda)^{-1} K_{m}(\vec{a}, \vec{b}, z) C_{\vec{a}}(z, \lambda) .
$$

This equation shows that the matrix function of the left side which is analytic (as a function of $\lambda$ ) on the set (1.9.1) is equivalent to a constant matrix, i.e. the eigenvalues of $K_{m}(\vec{a}, \vec{b}, \lambda)$ are independent of $\lambda$. If $c$ is such an eigenvalue then there exists a solution $\left(u_{1}, u_{2}\right)$ of $(1.11 .2)_{\vec{a}}$ at $z$ such that

$$
\left(u_{1}, u_{2}\right) K_{m}(\vec{a}, \vec{b}, \lambda)=c\left(u_{1}, u_{2}\right) \text {. }
$$

If $K_{m} \neq c I_{2}$ then we may conclude that $u_{2} / u_{1}$ may be continued as a meromorphic function throughout the set (1.9.1). This would be in particular true if $\left(u_{1}, u_{2}\right)$ were the unique bounded solution on $D(z, 1)$. This possibility of contradicting the existence of supersingular disks [3, Theorem 16.11] shows that $K_{m}(\vec{a}, \vec{b}, \lambda)$ is a scalar function whenever

$$
\left\{\begin{array}{c}
(p-1)\left(a_{1}, a_{2}, a_{3}\right) \in \mathbf{Z}_{p}, \\
\text { either } \mu_{3}>\operatorname{Max}\left(\mu_{1}, \mu_{2}\right) \text { or } \mu_{3}<\operatorname{Min}\left(\mu_{1}, \mu_{2}\right) .
\end{array}\right.
$$

Translating $\vec{b}$ by $\mathbf{Z}^{3}$ does not affect these conditions and so using analyticity of $K_{m}$ as a function of $\vec{b}$ with $\vec{\mu}$ fixed permits us to deduce that $K_{m}(\vec{a}, \vec{b}, \lambda)$ is a scalar function of $\lambda$ whenever $\mu_{3}$ does not lie between $\mu_{1}$ and $\mu_{2}$. It then follows from (2.11.6) that it is independent of $\lambda$.

REMARK. We do not believe that the quotient $u_{2} / u_{1}$ of components of a solution of (1.11.2) can be meromorphic on the set (1.9.1) except in the case in which $u_{2} / u_{1}$ is a rational function.

3. Variation of lifting of Frobenius. In this section we shall assume that $\vec{a}, \vec{b}, \lambda$ satisfy conditions (1.1), (1.4) and (1.9.1).

3.1. We observe that in the definitions (1.5) and (1.6) a choice has been made of $\lambda \rightarrow \lambda^{p}$ as a lifting of Frobenius for the parameter $\lambda$. We now generalize our formulation by considering a function $\lambda \rightarrow \phi(\lambda)$ analytic in a region of the type

$$
\operatorname{Sup}\left\{|\lambda|,\left|\lambda^{-1}\right|,|1-\lambda|^{-1}\right\}<1+\varepsilon
$$

for some $\varepsilon>0$ and satisfying the further condition that

$$
\left|\phi(\lambda)-\lambda^{p}\right|<1
$$


for all $\lambda$ satisfying (1.9.1). We observe that by (1.12.1)

$$
X_{\vec{b}}(x, \phi(\lambda))=C_{\vec{b}}\left(\lambda^{p}, \phi(\lambda)\right)^{t} X_{\vec{b}}\left(x, \lambda^{p}\right)
$$

which reminds us that the space $\omega_{\vec{b}, \lambda^{p}} L_{\lambda^{p}}$ coincides with $\omega_{\vec{b}, \phi(\lambda)} L_{\lambda^{p}}$ and that $L_{\lambda^{p}}=L_{\phi(\lambda)}$. The mapping $\psi_{1}$ (equation (1.5)) may also be described in terms of $\phi$, by means of the formula

$$
\psi_{1}\left(\xi x f_{\bar{a}}(x, \lambda) \frac{d x}{x}\right)=\psi_{0}\left(\xi F_{\vec{a}, b}(x, \lambda, \phi(\lambda))\right) \cdot x f_{\bar{b}}(x, \phi(\lambda)) \frac{d x}{x},
$$

where

$$
F_{\vec{a}, \vec{b}}(x ; \lambda, \phi(\lambda))=\frac{f_{\vec{a}}(x, \lambda)}{f_{\vec{b}}\left(x^{p}, \phi(\lambda)\right)}
$$

which is to be understood again as that element of $L_{\lambda}$ representing the indicated algebraic function of $x$ which for generic $x$ is approximated by (1.5.1). It will be convenient to explicitly denote the matrix of $\psi_{1}$ relative to this new basis of $\omega_{\vec{b}, \lambda^{p}} L_{\lambda^{p}} / d\left(f_{\vec{b}}\left(x, \lambda^{p}\right) L_{\lambda^{p}}\right)$ by writing

$$
\psi_{1} X_{\vec{a}}(x, \lambda)=\gamma(\vec{a}, \vec{b}, \lambda, \phi(\lambda)) X_{\vec{b}}(x, \phi(\lambda)) \bmod d\left(f_{\vec{b}}\left(x, \lambda^{p}\right) L_{\lambda^{p}}\right) .
$$

In particular, $\gamma(\vec{a}, \vec{b}, \lambda)=\gamma\left(\vec{a}, \vec{b}, \lambda, \lambda^{p}\right)$ while in general

$$
\gamma(\vec{a}, \vec{b}, \lambda, \phi(\lambda))=\gamma(\vec{a}, \vec{b}, \lambda) C_{\vec{b}}\left(\phi(\lambda), \lambda^{p}\right)^{t} .
$$

3.2. We now observe that in definitions (1.5) and (1.5.2) we have made a choice $x \mapsto x^{p}$ of lifting of Frobenius for the variable $x$. To generalize these definitions, let $\tau$ be an element of $L_{\lambda}$ such that

$$
\left|\tau(x)-x^{p}\right| \leqslant|p|
$$

for generic $x$ (and hence for all $x$ such that

$$
|x|=1=|x-1|=|1-x \lambda|) .
$$

We define

$$
F_{\vec{a}, \vec{b}}(x, \tau(x) ; \lambda)=\frac{f_{\vec{a}}(x, \lambda)}{f_{\vec{b}}\left(\tau(x), \lambda^{p}\right)}
$$

to be the unique element of $L_{\lambda}$ which represents the indicated function obviously algebraic over $L_{\lambda}$ and which for generic $x$ is approximated by (1.5.1). We define a mapping $\psi_{\tau, 0}$ of $L_{\lambda}$ into $L_{\lambda^{p}}$ by

$$
\left(\psi_{\tau, 0} \xi\right)(x)=\sum_{\tau(y)=x} \xi(y) / \tau^{\prime}(y),
$$

the sum being over the $p$ values of $y$ satisfying $\tau(y)=x$ such that $y$ is approximately a $p$ th root of $x$.

It follows from (3.2.1) that the approximate factorization of $\tau(y)-x$ represented by the congruence

$$
\tau(y)-x \equiv y^{p}-x \bmod p
$$


may be replaced by a precise factorization defined over $L_{\lambda^{p}}[3$, Chapter 20], i.e. there exists a unitary polynomial $\hat{\tau}(x, y)$ of degree $p$ in $y$ and with coefficients which are functions of $x$ in $L_{\lambda^{p}}$ such that for $x$ satisfying $|x|=1=|x-1|=\left|1-x \lambda^{p}\right|$, the roots of the equation $\tau(y)=x$ allowed in the sum in the definition (3.2.4) are precisely the roots of the polynomial equation $\hat{\tau}(x, y)=0$. Furthermore

$$
\hat{\tau}(x, y) \equiv y^{p}-x \bmod p .
$$

Thus in (3.2.4) we may write

$$
\left(\psi_{\tau, 0} \xi\right)(x)=\sum_{y \mid \hat{\tau}(x, y)=0} \xi(y) / \tau^{\prime}(y)
$$

We now define $\psi_{\tau, 1}$, a mapping of $\omega_{\vec{a}, \lambda} L_{\lambda}$ into $\omega_{\vec{b}, \lambda^{p}} L_{\lambda^{p}}$, by setting for $\xi \in L_{\lambda^{p}}$,

$$
\psi_{\tau, 1}\left(\xi f_{\vec{a}}(x, \lambda) d x\right)=\psi_{\tau, 0}\left(\xi F_{\vec{a}, \vec{b}}(x, \tau(x) ; \lambda)\right) f_{\vec{b}}\left(x, \lambda^{p}\right) d x .
$$

We show $\psi_{\tau, 1}$ and $\psi_{1}$ are essentially the same on $\omega_{\vec{a}, \lambda} L_{\lambda}$.

LeMma 3.3. $\psi_{\tau, 1}-\psi_{1}$ maps $\omega_{\vec{a}, \lambda}$ into $d\left(L_{\lambda^{p}} f_{\vec{b}}\left(x, \lambda^{p}\right)\right)$.

Proof. Let $\xi \in L_{\lambda}$; define

$$
\Delta=\psi_{\tau, 0} \xi F_{\vec{a}, \vec{b}}(x, \tau(x), \lambda)-\psi_{0} \xi F_{\vec{a}, \vec{b}}\left(x, \lambda^{p}\right) .
$$

We assert that

$$
\Delta \in \frac{1}{f_{b}\left(x, \lambda^{p}\right)} \frac{d}{d x} L_{\lambda^{p}} f_{\vec{b}}\left(x, \lambda^{p}\right) .
$$

This would be enough to prove the assertion. We first make a local calculation, say with $x$ in the generic disk,

$$
\left\{\begin{array}{l}
\hat{\tau}(x, y)=0 \quad(\text { hence } \tau(y)=x) \\
x=\bar{y}^{p}
\end{array}\right.
$$

and letting $f_{\vec{a}}(\bar{y}, \lambda), f_{\vec{b}}\left(x, \lambda^{p}\right)$ denote branches of $f_{\vec{a}}$ and $f_{\vec{b}}$ which are compatible in the sense that their ratio is $F_{\vec{a}, \vec{b}}(\bar{y}, \lambda)$. We find

$$
\Delta=\sum_{y} \xi(y) \frac{f_{\vec{a}}(y, \lambda)}{f_{\vec{b}}\left(x, \lambda^{p}\right)} \frac{1}{\tau^{\prime}(y)}-\sum_{\bar{y}} \xi(\bar{y}) \frac{f_{\vec{a}}(\bar{y}, \lambda)}{f_{\vec{b}}\left(x, \lambda^{p}\right)} \frac{1}{p \bar{y}^{p-1}}
$$

each of the sums being over solutions for $y$ (resp. $\bar{y}$ ) of (3.3.2). By that equation

$$
\frac{d y}{d x}=\frac{1}{\tau^{\prime}(y)}, \quad \frac{d \bar{y}}{d x}=\frac{1}{p \bar{y}^{p-1}}
$$

and so letting $(y, \bar{y})$ run over all pairs of solutions of (3.3.2) we obtain

$$
p \Delta=\frac{1}{f_{\vec{b}}\left(x, \lambda^{p}\right)} \sum_{y, \bar{y}} \xi(y) f_{\vec{a}}(y, \lambda) \frac{d y}{d x}-\xi(\bar{y}) f_{\vec{a}}(\bar{y}, \lambda) \frac{d \bar{y}}{d x} .
$$

Let us write $G(y)=\xi(y) f_{\vec{a}}(y, \lambda)$ which as a function of $y$ is bounded on the generic disk. Hence

$$
\left|\frac{1}{i !}\right|\left|G^{(i)}(y)\right|<|\xi|_{\text {gauss }}
$$


and so we may compute

$$
G(y) \frac{d y}{d x}-G(\bar{y}) \frac{d \bar{y}}{d x}=\frac{d}{d x} \sum_{i=0} G^{(i)}(\bar{y}) \rho^{i+1} /(i+1) !,
$$

where

$$
\rho=y-\bar{y}, \quad|\rho|<1 .
$$

Using the definition of $G$, we find

$$
\begin{aligned}
\sum_{i=0} G^{(i)}(\bar{y}) \rho^{i+1} /(i+1) & =\sum_{i=0} \frac{1}{i+1} \rho^{i+1} \sum_{l+k=i} \frac{1}{l !} \xi^{(l)}(\bar{y}) \frac{1}{k !} f_{\bar{a}}^{(k)}(\bar{y}, \lambda) \\
& =f_{\bar{b}}\left(x, \lambda^{p}\right) \vec{F}_{, \bar{b}}(\bar{y}, \lambda) \eta(y, \bar{y})
\end{aligned}
$$

where

$$
\eta(y, \bar{y})=\sum_{i=0} \frac{(y-\bar{y})^{i+1}}{i+1} \sum_{l+k=i} \frac{1}{l !} \xi^{(l)}(\bar{y}) \frac{1}{k !} \frac{f_{\vec{a}}^{(k)}(\bar{y}, \lambda)}{f_{\vec{a}}(\vec{y}, \lambda)} .
$$

For $g \in L_{\lambda}$ we may write uniquely

$$
g(\bar{y})=A_{0}(x)+A_{1}(x) \bar{y}+\cdots+A_{p-1}(x) \bar{y}^{\rho-1},
$$

where each $A_{i}$ lies in $L_{\lambda^{p}}$ and $p|g|_{\text {gauss }} \geqslant\left|A_{i}\right|_{\text {gauss }}$. Indeed

$$
p A_{0}(x)=\sum_{\bar{y}^{p}=x} g(\bar{y})
$$

and similarly for the remaining $A_{i}$. This shows that

$$
\eta(y, \bar{y})=\sum_{i=0} \frac{(y-\bar{y})^{i+1}}{i+1} \sum_{j=0}^{p-1} A_{i, j}(x) \bar{y}^{j},
$$

where each $A_{i, j}$ lies in $L_{\lambda}$ with uniformly bounded gauss norm. Using relation (3.2.4.1), we deduce

$$
(y-\bar{y})^{p}=p B(x, y, \bar{y}),
$$

where $B$ is a polynomial in $y, \bar{y}$ of degree bounded by $p-1$ in each variable with coefficients lying in $L_{\lambda^{p}}$ and gauss norm bounded by unity. Since $p^{[m / p]} / m \rightarrow 0$ in $\mathbf{Q}_{p}$ as $m \rightarrow \infty$, it follows that

$$
\eta(y, \bar{y})=\sum_{\substack{i<p \\ j<p}} \alpha_{i, j}(x) y^{i} \bar{y}^{j},
$$

where each $\alpha_{i, j}$ lies in $L_{\lambda^{p}}$. A similar result holds for the product $F_{\bar{a}, \bar{b}}(\bar{y}, \lambda) \eta(y, \bar{y})$.

By (3.3.4)

$$
p \Delta=\frac{1}{f_{b}\left(x, \lambda^{p}\right)} \frac{d}{d x}\left(f_{\vec{b}}\left(x, \lambda^{p}\right) J(x)\right),
$$

where $J(x)=\sum_{y, \bar{y}} F_{\bar{a}, \bar{b}}(\bar{y}, \lambda) \eta(y, \bar{y})$. It follows from the theory of symmetric function that $J \in L_{\lambda^{p}}$. This completes the proof of (3.3.1) and hence of the lemma.

4. Transformation of $\gamma(a, b, \lambda)$ under the Kummer group. The object of this section is to show that for $\vec{a}, \vec{b}, \lambda$ satisfying (1.1), (1.4) and (1.9) we have $K_{m}(\vec{a}, \vec{b}, \lambda)$ $=I_{2}$. We make this explicit. 
THEOREM. Subject to the above hypotheses, for $1 \leqslant m \leqslant 24$ with $h_{m}, N_{m}, M_{m}$ as given in Table II and (2.6),

$$
h_{m}(\vec{a}, \vec{b}, \lambda) \gamma\left(M_{m}(\vec{a}), M_{m}(\vec{b}) ; \theta_{m}(\lambda), \theta_{m}\left(\lambda^{p}\right)\right)=N_{m}(\lambda)^{-1} \gamma(\vec{a}, \vec{b}, \lambda) N_{m}\left(\lambda^{p}\right) .
$$

Proof. We substitute $x=T_{m}(t, \lambda)$ in (1.6) and obtain from (2.3)

$$
\begin{aligned}
& \psi_{1, \tau}\left(H_{m}(\vec{a}, \lambda) X_{M_{m}(\vec{a})}\left(t, \theta_{m}(\lambda)\right)\right) \\
& \quad \equiv \gamma(\vec{a}, \vec{b}, \lambda) H_{m}^{\sigma}\left(\vec{b}, \lambda^{p}\right) X_{M_{m}(\vec{b})}\left(t, \theta_{m}\left(\lambda^{p}\right)\right) \quad \text { mod exact. }
\end{aligned}
$$

The only point that needs clarification is the choice of branches of the transformed algebraic functions. We know that $f_{\vec{a}}(x, \lambda)$ and $f_{\vec{b}}\left(\tau(x), \lambda^{p}\right)$ must be interpreted as branches whose ratio is approximated by (1.5.1). The same must hold for their images under $T_{m}(t, \lambda)$. Thus for example for $m=3$ computing formally ( $t=1-x$ ) we obtain

$$
\begin{aligned}
f_{\vec{a}}(x, \lambda) & =(1-t)^{a_{2}-1}(t)^{a_{3}-a_{2}}(1-\lambda(1-t))^{-a_{1}} \\
& =(1-\lambda)^{-a_{1}} t^{a_{3}-a_{2}}(1-t)^{a_{2}-1}\left(1-\theta_{3}(\lambda) t\right)^{-a_{1}} .
\end{aligned}
$$

We may choose $\tau(x)=1-t^{p}$ and so we have formally

$$
f_{\vec{b}}\left(\tau(x), \lambda^{p}\right)=\left(1-\lambda^{p}\right)^{-b_{1}} t^{p\left(b_{3}-b_{2}\right)}\left(1-t^{p}\right)^{b_{2}-1}\left(1-\theta_{3}\left(\lambda^{p}\right) t^{p}\right)^{-b_{1}}
$$

and now we must interpret (4.3) and (4.2) so that (1.5.1) holds. This will indeed be the case if we do the following:

$$
\begin{aligned}
& \text { interpret }(1-\lambda)^{-a_{1}},\left(1-\lambda^{p}\right)^{-b_{1}} \text { so that } \frac{(1-\lambda)^{-a_{1}}}{\left(1-\lambda^{p}\right)^{-b_{1}}} \equiv(1-\lambda)^{\mu_{1}}, \\
& \text { interpret } f_{a_{1}, a_{3}-a_{2}+1, a_{3}}\left(t, \theta_{3}(\lambda)\right) \text { and } f_{b_{1}, b_{3}-b_{2}+1, b_{3}}\left(t^{p}, \theta_{3}\left(\lambda^{p}\right)\right)
\end{aligned}
$$

so that the ratio is approximated by the rational function deduced from (1.5.1) with $\vec{a}, \vec{b}$ replaced by $\left(a_{1}, a_{3}-a_{2}+1, a_{3}\right),\left(b_{1}, b_{3}-b_{2}+1, b_{3}\right)$ and $x, \lambda$ replaced by $\left(t, \theta_{3}(\lambda)\right)$. In this way we deduce

$$
\begin{aligned}
& h_{m}(\vec{a}, \vec{b} ; \lambda) N_{m}(\lambda) \psi_{1}\left(X_{M_{m}(\vec{a})}\left(t, \theta_{m}(\lambda)\right)\right) \\
& \quad=\gamma(\vec{a}, \vec{b}, \lambda) N_{m}\left(\lambda^{p}\right) X_{M_{m}(\vec{b})}\left(t, \theta_{m}\left(\lambda^{p}\right)\right) \quad \text { mod exact. }
\end{aligned}
$$

By (3.1.6)

$$
\begin{aligned}
& \psi_{1}\left(X_{M_{m}(\vec{a})}\left(t, \theta_{m}(\lambda)\right)\right) \\
& \quad \equiv \gamma\left(M_{m}(\vec{a}), M_{m}(\vec{b}) ; \theta_{m}(\lambda), \theta_{m}\left(\lambda^{p}\right)\right) X_{M_{m}(\vec{b})}\left(t, \theta_{m}\left(\lambda^{p}\right)\right) \quad \text { mod exact }
\end{aligned}
$$

This completes the proof for $m=3$. The other cases may be checked similarly. We observe, however, that strictly speaking it is not necessary to check each $m$. The group of $M_{m}$ mod translations by $\mathbf{Z}^{3}$ is in fact the octahedral group [2, p. 408] and is generated by $M_{13}$ and $M_{7}\left(M_{13}\right.$ is of order two, $M_{7}$ is of order $3, M_{21}$ is of order 4 
with a relation $M_{21} M_{7} M_{13}=I_{3}$ ). Since $M_{9} M_{3}=M_{7}$, the group is generated by $M_{3}$, $M_{9}, M_{13}$. The transformations $T_{9}$ and $T_{13}$ are relatively simple to handle and so the transformation law for $M_{3}$ is the most interesting. This completes our treatment of the theorem.

5. Twist transformation. We now find the relation between $\gamma(\vec{a}, \vec{b}, \lambda)$ and $\gamma\left(\vec{a}^{t w}, \vec{b}^{t w}, \lambda\right)$, where $\vec{a}^{t w}=\left(a_{2}, a_{1}, a_{3}\right)$. As usual we shall assume conditions (1.1), (1.4) and (1.9.1) to be satisfied.

\section{TheOREM.}

$$
\gamma\left(\vec{a}^{t w}, \vec{b}^{t w}, \lambda\right) \zeta(\vec{a}, \vec{b})=\left(\begin{array}{rc}
a_{3}-a_{1}, & 0 \\
0, & a_{3}-a_{2}
\end{array}\right) \gamma(\vec{a}, \vec{b}, \lambda)\left(\begin{array}{rc}
b_{3}-b_{1}, & 0 \\
0, & b_{3}-b_{2}
\end{array}\right)^{-1},
$$

where

$$
\zeta(\vec{a}, \vec{b})=p \frac{\gamma_{p}\left(1+a_{3}-a_{2}, 1+b_{3}-b_{2}\right)}{\gamma_{p}\left(1-a_{2}, 1-b_{2}\right) \gamma_{p}\left(a_{1}, b_{1}\right) \gamma_{p}\left(a_{3}-a_{1}, b_{3}-b_{1}\right)(-1)^{\mu_{2}}}
$$

is a scalar function of $\vec{a}, \vec{b}$, independent of $\lambda$.

Proof. By duality [4, (3.22.7)]

$$
\begin{gathered}
\gamma(\vec{a}, \vec{b}, \gamma)^{t}\left(\begin{array}{cc}
\left(a_{3}-a_{1}\right) \lambda, & 0 \\
0, & \left(a_{3}-a_{2}\right)(1-\lambda)
\end{array}\right) \gamma(\kappa(\vec{a}), \kappa(\vec{b}), \lambda) \\
=p\left(\begin{array}{cc}
\left(b_{3}-b_{1}\right) \lambda^{p}, & 0 \\
0, & \left(b_{3}-b_{2}\right)\left(1-\lambda^{p}\right)
\end{array}\right),
\end{gathered}
$$

where $\kappa(\vec{a})=\left(1-a_{1}, 1-a_{2}, 1-a_{3}\right)$. We observe that $M_{18}\left(\vec{a}^{t w^{\prime}}\right)=\kappa(\vec{a})$, while $h_{18}(\vec{a}, \vec{b}, \lambda)$ is unchanged under $(\vec{a}, \vec{b}) \rightarrow\left(\vec{a}^{t w}, \vec{b}^{t w}\right)$. Thus

$$
\left(\begin{array}{cc}
0, & 1-\lambda \\
-\lambda, & 0
\end{array}\right) \gamma(\kappa(\vec{a}), \kappa(\vec{b}), \lambda)=\gamma\left(\vec{a}^{t w}, \vec{b}^{t w}, \lambda\right)\left(\begin{array}{cc}
0, & 1-\lambda^{p} \\
-\lambda^{p}, & 0
\end{array}\right) \frac{1}{\hat{h}_{18}(\vec{a}, \vec{b}, \lambda)},
$$

where $\hat{h}_{18}(\vec{a}, \vec{b}, \lambda)=h_{18}(\vec{a}, \vec{b} ; \lambda)\left(1-\lambda^{p}\right) /(1-\lambda)$. Substituting in this equation gives

$$
\begin{gathered}
\gamma(\vec{a}, \vec{b}, \lambda)^{t}\left(\begin{array}{cc}
0, & -\left(a_{3}-a_{1}\right) \\
a_{3}-a_{2}, & 0
\end{array}\right) \gamma\left(\vec{a}^{t w}, \vec{b}^{t w}, \lambda\right) \\
=p \hat{h}_{18}(\vec{a}, \vec{b}, \lambda)\left(\begin{array}{cc}
0, & -\left(b_{3}-b_{1}\right) \\
b_{3}-b_{2}, & 0
\end{array}\right) .
\end{gathered}
$$

We multiply the right by $\gamma\left(\vec{a}^{t w}, \vec{b}^{t w}, \lambda\right)^{-1}$, take the transpose, multiply on the left by $\left(\begin{array}{cc}0 & -1 \\ 1 & 0\end{array}\right)$ and deduce

(5.4) right side of $(5.0)=p \hat{h}_{18}(\vec{a}, \vec{b}, \lambda)\left(\begin{array}{rr}0 & -1 \\ 1 & 0\end{array}\right) \gamma\left(\vec{a}^{t \omega}, \vec{b}^{t \omega}, \lambda\right) *\left(\begin{array}{rr}0 & 1 \\ -1 & 0\end{array}\right)$. 
If $X$ is a $2 \times 2$ matrix then

$$
\left(\begin{array}{rr}
0, & -1 \\
1, & 0
\end{array}\right) \operatorname{det} X=X\left(\begin{array}{rr}
0, & -1 \\
1, & 0
\end{array}\right) X^{t}
$$

and so the right side of (5.4) is

$$
p \hat{h}_{18}(\vec{a}, \vec{b}, \lambda) \gamma\left(\vec{a}^{t w}, \vec{b}^{t w}, \lambda\right) \cdot \frac{1}{\operatorname{det} \gamma\left(\vec{a}^{t w}, \vec{b}^{t w}, \lambda\right)} .
$$

Thus (5.0) is valid if $\zeta$ is the scalar function

$$
\zeta=p \hat{h}_{18}(\vec{a}, \vec{b}, \lambda) / \operatorname{det} \gamma\left(\vec{a}^{t w}, \vec{b}^{t w}, \lambda\right) .
$$

Now by means of [3, Theorem 25]

(5.8) $\operatorname{det} \gamma\left(\vec{a}^{t w}, \vec{b}^{t w}, \lambda\right)=\lambda^{\mu_{3}} \frac{\left(1-\lambda^{p}\right)^{b_{1}+b_{2}-b_{3}}}{(1-\lambda)^{a_{1}+a_{2}-a_{3}}}(-1)^{\mu_{1}-\mu_{3}}$

$$
\times \frac{\gamma_{p}\left(1-a_{2}, 1-b_{2}\right) \gamma_{p}\left(a_{1}, b_{1}\right) \gamma_{p}\left(a_{3}-a_{1}, b_{3}-b_{1}\right)}{\gamma_{p}\left(1+a_{3}-a_{2}, 1+b_{3}-b_{2}\right)}
$$

On the other hand, by (2.6)

$$
\hat{h}_{18}(\vec{a}, \vec{b}, \lambda)=(-1)^{\mu_{3}-\mu_{1}-\mu_{2}} \lambda^{\mu_{3}} \frac{(1-\lambda)^{a_{3}-a_{1}-a_{2}}}{\left(1-\lambda^{p}\right)^{b_{3}-b_{1}-b_{2}}} .
$$

This completes the proof of the theorem.

5.10. We now give an independent treatment of the theorem based purely on the differential equation point of view. We shall assume $a_{1} \notin \mathbf{Z}$. This will put us in the nonlogarithmic case for the solutions of zero. There is a similar and indeed easier treatment of the logarithmic case. Let us write (cf. [3, Lemma 24.1])

$$
\begin{aligned}
& V_{\vec{a}}(\lambda)=\left(\begin{array}{l}
\left(a_{3}-a_{2}\right) F\left(a_{1}, a_{2}, 1+a_{3} ; \lambda\right) \\
\left(1-a_{3}\right) F\left(a_{1}-a_{3}, a_{2}-a_{3}, 1-a_{3} ; \lambda\right) \\
a_{3} F\left(a_{1}, a_{2}, a_{3} ; \lambda\right) \\
\left(a_{3}-a_{1}\right) \lambda F\left(a_{1}+1-a_{3}, a_{2}+1-a_{3}, 2-a_{3} ; \lambda\right)
\end{array}\right) .
\end{aligned}
$$

Then by [3, Lemma 24.3]

$$
\begin{gathered}
V_{\vec{b}}\left(\lambda^{p}\right) \gamma(\vec{a}, \vec{b}, \lambda)^{t}=\Delta(\vec{a}, \vec{b}, \lambda) V_{\vec{a}}(\lambda), \\
V_{\vec{b}^{t \omega}}\left(\lambda^{p}\right) \gamma\left(\vec{a}^{t \omega}, \vec{b}^{t w}, \lambda\right)^{t}=\Delta\left(\vec{a}^{t \omega}, \vec{b}^{t w}, \lambda\right) V_{\vec{a}^{t w}}(\lambda),
\end{gathered}
$$

where $\Delta(\vec{a}, \vec{b}, \lambda)$ is a diagonal matrix given explicitly in this nonlogarithmic case [3, Theorem 25] by

$$
\Delta(\vec{a}, \vec{b}, \lambda)=\left(\begin{array}{cc}
\theta_{1}(\vec{a}, \vec{b}) & 0 \\
0 & \lambda^{\mu_{3}} \theta_{4}(\vec{a}, \vec{b})
\end{array}\right)
$$


where

$$
\begin{gathered}
\theta_{1}(a, b)=\frac{\gamma_{p}\left(a_{2}, b_{2}\right) \gamma_{p}\left(a_{3}-a_{2}, b_{3}-b_{2}\right)}{\gamma_{p}\left(1+a_{3}, 1+b_{3}\right)}, \\
\theta_{4}(\vec{a}, \vec{b})=(-1)^{\mu_{2}-\mu_{3}} \frac{\gamma_{p}\left(a_{3}-1, b_{3}-1\right) \gamma_{p}\left(1-a_{1}, 1-b_{1}\right)}{\gamma_{p}\left(1+a_{3}-a_{1}, 1+b_{3}-b_{1}\right)} .
\end{gathered}
$$

It is easily deduced from (5.10.1) that

$$
V_{\vec{a}^{\prime k}}=\left(\begin{array}{cc}
a_{3}-a_{2} & 0 \\
0 & a_{3}-a_{1}
\end{array}\right)^{-1} V_{\vec{a}}\left(\begin{array}{cc}
a_{3}-a_{1} & 0 \\
0 & a_{3}-a_{2}
\end{array}\right)
$$

and similarly for $V_{\vec{b}^{\prime *}}$ in terms of $V_{\vec{b}}$. Substituting these in (5.10.3) we find

$$
\begin{aligned}
& \left(\begin{array}{cc}
b_{3}-b_{1} & 0 \\
0 & b_{3}-b_{2}
\end{array}\right) \gamma\left(\vec{a}^{t w}, \vec{b}^{t w}, \lambda\right)^{t}\left(\begin{array}{ll}
a_{3}-a_{1} & \\
\quad & a_{3}-a_{2}
\end{array}\right)^{-1} \\
& \quad=V_{\vec{b}}\left(\lambda^{p}\right)^{-1}\left(\begin{array}{cc}
b_{3}-b_{2} & 0 \\
0 & b_{3}-b_{1}
\end{array}\right) \Delta\left(\vec{a}^{t w}, \vec{b}^{t w}, \lambda\right)\left(\begin{array}{cc}
a_{3}-a_{2} & \\
& a_{3}-a_{1}
\end{array}\right)^{-1} V_{\vec{a}}(\lambda) .
\end{aligned}
$$

From this the proof of the theorem is reduced to verifying

$$
\zeta(\vec{a}, \vec{b})\left(\begin{array}{cc}
b_{3}-b_{2} & 0 \\
0 & b_{3}-b_{1}
\end{array}\right) \Delta\left(\vec{a}^{t w}, \vec{b}^{t w}, \lambda\right)\left(\begin{array}{cc}
a_{3}-a_{2} & 0 \\
0 & a_{3}-a_{1}
\end{array}\right)^{-1}=\Delta(\vec{a}, \vec{b}, \lambda) .
$$

This verification follows easily from the explicit formulae (5.10.4) and (5.10.5).

In the logarithmic case $\left(a_{3}=b_{3}=1\right)$ we put

$$
V_{\vec{a}}(\lambda)=\left(\begin{array}{cc}
\left(1-a_{2}\right) F\left(a_{1}, a_{2}, 2, \lambda\right) & F\left(a_{1}, a_{2}, 1, \lambda\right) \\
\left(1-a_{2}\right) H\left(a_{1}, a_{2}, 2, \lambda\right) & H\left(a_{1}, a_{2}, 1, \lambda\right)
\end{array}\right),
$$

where for $n \in \mathbf{N}, n \geqslant 1, H\left(a_{1}, a_{2}, n, \lambda\right)$ is defined by the conditions that it lie in $\mathbf{Q}\left(a_{1}, a_{2}\right)((\lambda))$ and that

$$
y=H\left(a_{1}, a_{2}, n, \lambda\right)+F\left(a_{1}, a_{2}, n, \lambda\right) \log \lambda
$$

be a formal solution of $\left(\lambda\left(\delta+a_{1}\right)\left(\delta+a_{2}\right)-\delta(\delta+n-1)\right) y=0$, where $\delta=$ $\lambda d / d \lambda$.

Equation (5.10.2) now takes the form

$$
V_{\vec{b}}\left(\lambda^{p}\right) \gamma(\vec{a}, \vec{b}, \lambda)^{t}=\theta\left(\begin{array}{ll}
1 & 0 \\
\nu & p
\end{array}\right) V_{\vec{a}}(\lambda)
$$

where [3, Theorem 2.6.1]

$$
\theta=(-1)^{\mu_{2}}, \quad \frac{\nu}{p}=\frac{\Gamma_{p}^{\prime}}{\Gamma_{p}}\left(a_{1}\right)+\frac{\Gamma_{p}^{\prime}}{\Gamma_{p}}\left(a_{2}\right)-2 \frac{\Gamma_{p}^{\prime}}{\Gamma_{p}}(1)
$$

The second verification of the theorem in this case causes no difficulty.

6. Hypergeometric-Boyarsky gamma function. Up to this point we have studied the transformation laws of $\gamma(\vec{a}, \vec{b} ; \lambda)$ subject to conditions (1.1), (1.4) and (1.9.1). 
We now recall the hypergeometric Boyarsky gamma function. Let

$$
\begin{aligned}
S^{\prime} & =\left\{\vec{z}=\left(z_{1}, z_{2}, z_{3}\right) \mid \text { ord } z_{i}>-e_{2}, i=1,2,3\right\}, \\
S & =\left\{\vec{x}=-\vec{\mu}+p \vec{z} \mid z \in S^{\prime}, 0 \leqslant \mu_{1}<p, \mu_{i} \in \mathbf{N}, i=1,2,3\right\} .
\end{aligned}
$$

We define [4, §3.19, Theorem 3.22] $\Gamma_{B}(\vec{x}, \lambda)$ for $\vec{x} \in S$ and $\lambda$ satisfying (1.9.1) by the condition that it is an analytic function of $\vec{x}$ (for $\vec{\mu}$ fixed) and that

$$
\Gamma_{B}(\vec{a}, \lambda)=\gamma(\vec{a}, \vec{b}, \lambda)
$$

for all $\vec{a}$ satisfying (1.1) with $\vec{b}$ chosen uniquely so that (1.4) holds with

$$
\mu_{1}, \mu_{2}, \mu_{3} \text { in }[0, p-1] \text {. }
$$

We also recall $[4,(3.2)]$ the change of basis formula for $\vec{a}, \vec{b}$ satisfying (1.1), (1.4), $m_{1}, m_{2}, m_{3} n_{1}, n_{2}, n_{3}$ all in $\mathbf{Z}$.

$$
\begin{aligned}
\gamma(\vec{a} & \left.+\left(m_{1}, m_{2}, m_{3}\right), \vec{b}+\left(n_{1}, n_{2}, n_{3}\right), \lambda\right) \\
& =B\left(\vec{a}, \vec{a}+\left(m_{1}, m_{2}, m_{3}\right), \lambda\right) \gamma(\vec{a}, \vec{b} ; \lambda) B\left(\vec{b}, \vec{b}+\left(n_{1}, n_{2}, n_{3}\right), \lambda^{p}\right)^{-1},
\end{aligned}
$$

where the matrix $B$ is defined by (1.2).

THEOREM. In the notation of Table II, with $\vec{\mu}$ satisfying (6.3) let

$$
\hat{\mu}_{m}=-M_{m}(0)+\frac{1}{p}\left[M_{m}(-\vec{\mu})+\operatorname{Rep}\left(-M_{m}(-\vec{\mu})\right)\right],
$$

where Rep denotes the mapping of $\mathbf{Z}_{p}$ into $\{0,1, \ldots, p-1\}$ obtained by taking a representative of class modulo $p$. For $\lambda$ satisfying (1.9.1) and $\vec{x}=-\mu+p \vec{z}$ lying in $S$ $\left(\vec{z} \in S^{\prime}\right)$ we have

$$
\begin{aligned}
h_{m}(\vec{x}, \vec{z}, \lambda) \Gamma_{B}\left(M_{m}(\vec{x}), \theta_{m}(\lambda)\right) \\
=N_{m}(\lambda)^{-1} \Gamma_{B}(\vec{x}, \lambda) N_{m}\left(\lambda^{p}\right) C_{M_{m}(\vec{z})}\left(\theta_{m}(\lambda)^{p}, \theta_{m}\left(\lambda^{p}\right)\right)^{t} \\
\quad \times B\left(M_{m}(\vec{z}), M_{m}(\vec{z})+\hat{\mu}_{m}, \theta_{m}(\lambda)^{p}\right)^{-1} .
\end{aligned}
$$

Proof. We recall that $h_{m}(\vec{a}, \vec{b} ; \lambda)$ is a product of the form

$$
\lambda^{\mu_{1}} \times(1-\lambda)^{a_{i}} /\left(1-\lambda^{p}\right)^{b_{i}} .
$$

The second factor is of the form

$$
(1-\lambda)^{\mu_{i}}\left((1-\lambda)^{p} /\left(1-\lambda^{p}\right)\right)^{b_{i}}
$$

and so we conclude that $h_{m}(\vec{x}, \vec{z}, \lambda)$ is analytic, as a function of $\vec{x}, \lambda$ subject to the condition

$$
\operatorname{Inf}(0, \text { ord } \vec{z})+1>\frac{1}{p-1}+\operatorname{Sup}(0,(p-1) \operatorname{ord}(\lambda-1))
$$

and hence is analytic subject to (1.9.1) for $\vec{x}$ in $S$. Again subject to (1.9.1), $\theta_{m}(\lambda)$ cannot lie in $D\left(1,1^{-}\right)$and so by Lemma 1.13 the matrix $C_{M_{m}(\vec{z})}\left(\theta_{m}(\lambda)^{p}, \theta_{m}\left(\lambda^{p}\right)\right)$ is analytic for

$$
1+\text { ord } \vec{z}>\frac{1}{p-1}
$$


and hence surely for $\vec{x} \in S$. It only remains to verify (6.6) for $\vec{x}=\vec{a}=p \vec{b}-\vec{\mu}$ with $a, b, \vec{\mu}$ satisfying (1.1), (1.4) and (6.3). This follows from Theorem 4 using (3.1.7) to deduce

$$
\begin{aligned}
& \gamma\left(M_{m}(\vec{a}), M_{m}(\vec{b}) ; \theta_{m}(\lambda), \theta_{m}\left(\lambda^{p}\right)\right) \\
& \quad=\gamma\left(M_{m}(\vec{a}), M_{m}(\vec{b}) ; \theta_{m}(\lambda)\right) C_{M(\vec{b})}\left(\theta_{m}\left(\lambda^{p}\right), \theta_{m}(\lambda)^{p}\right)^{t}
\end{aligned}
$$

and using (6.4) to deduce

$$
\begin{gathered}
\gamma\left(M_{m}(\vec{a}), M_{m}(\vec{b}), \theta_{m}(\lambda)\right) B\left(M_{m}(\vec{b}), M_{m}(\vec{b})+\hat{\mu}_{m}, \theta_{m}(\lambda)^{p}\right)^{-1} \\
=\gamma\left(M_{m}(\vec{a}), M_{m}(\vec{b})+\hat{\mu}_{m}, \theta_{m}(\lambda)\right)=\Gamma_{B}\left(M_{m}(\vec{a}), \theta_{m}(\lambda)\right),
\end{gathered}
$$

the point being that $(6.5)$ implies

$$
p\left(M_{m}(\vec{b})+\hat{\mu}_{m}\right)=M_{m}(\vec{a})+\operatorname{Rep}-M_{m}(\vec{a}) .
$$

This completes the proof of the theorem.

6.12. Corollary. $\Gamma_{B}(\vec{x}, \lambda)$ is analytic in $\vec{x}$ and $\lambda$ for $x$ in $S$ and $\lambda \neq \infty$;

$$
\operatorname{ord}(\lambda-1)<\frac{1}{(p-1)}\left[1-\frac{1}{(p-1)}\left(2-\frac{1}{p-1}-\frac{1}{p}\right)\right] \text {. }
$$

Proof. This has been explained (subsection 1.10) for ord $(\lambda-1) \leqslant 0$. It only remains to remove this constraint. Using the theorem with $m=5$, we obtain

$$
\begin{aligned}
(-1)^{\mu_{2}} & \Gamma_{B}\left(x_{1}, x_{2}, x_{1}+x_{2}-x_{3} ;(1-\lambda)\right) \\
= & \left(\begin{array}{ll}
0, & 1 \\
1, & 0
\end{array}\right) \Gamma_{B}(\vec{x}, \lambda)\left(\begin{array}{rr}
0, & 1 \\
1, & 0
\end{array}\right) C_{\left(z_{1}, z_{2}, z_{1}+z_{2}-3\right)}\left((1-\lambda)^{p}, 1-\lambda^{p}\right)^{t} \\
& \cdot\left\{\begin{array}{r}
B\left(z_{1}, z_{2}, z_{1}+z_{2}-z_{3} ; z_{1}, z_{2}, z_{1}+z_{2}-z_{3}-1 ;(1-\lambda)^{p}\right)^{-1} \\
\text { if } \mu_{1}+\mu_{2} \geqslant \mu_{3}+p, p+\mu_{3}>\mu_{1}+\mu_{2} \geqslant \mu_{3}, \\
B\left(z_{1}, z_{2}, z_{1}+z_{2}-z_{3} ; z_{1}, z_{2}, z_{1}+z_{2}-z_{3}+1 ;(1-\lambda)^{p}\right)^{-1} \\
\text { if } \mu_{3}>\mu_{1}+\mu_{2}
\end{array}\right.
\end{aligned}
$$

valid for $\vec{x} \in S$ and $\lambda$ satisfying (1.9.1). The fact or $\Gamma_{B}(\vec{x}, \lambda)$ on the right may be extended to cover the disk $D\left(0,1^{-}\right)$except possibly for a pole at $\lambda=0$. Furthermore, the factor $C_{\left(z_{1}, z_{2}, z_{1}+z_{2}-z_{3}\right)}\left((1-\lambda)^{p}, 1-\lambda^{p}\right)$ is by Lemma 1.13 analytic as a function of $\vec{z}, \lambda$ in the region

$$
\operatorname{Inf}(0, \operatorname{ord} \vec{z})-\operatorname{ord} \lambda^{p}+\operatorname{ord}\left((1-\lambda)-(1-\lambda)^{p}\right)>\frac{1}{p-1} .
$$

(In this argument we may assume $|\lambda|<1$.) Since ord $\vec{z}>-e_{2}$ and

$$
\operatorname{ord}\left(\left(1-\lambda^{p}\right)-(1-\lambda)^{p}\right) \geqslant 1+\operatorname{ord} \lambda,
$$

condition (6.12.2) becomes

$$
1-e_{2}-(p-1) \text { ord } \lambda>\frac{1}{p-1} .
$$


The corollary now follows, remembering to replace $\lambda$ by $1-\lambda$, and observing that the possible singular locus introduced by the matrix $B$ in (6.12.1) may be ruled out (for example) by comparison with (6.6) with $m=3$.

6.13. CoROlLaRY. The transformation formula (6.6) remains valid for $\vec{x}$ in $S$ and condition (1.9.1) replaced by

$$
\operatorname{Inf}(|\lambda|,|1-\lambda|,|1 / \lambda|)>1-\varepsilon
$$

for some real number $\varepsilon>0$.

PROOF. The assertion follows from analytic continuation and Corollary 6.12 .

6.14. CoRollary. For $x \in \vec{S}$ and $\lambda$ satisfying (6.13.1),

$\Gamma_{B}\left(\left(x_{2}, x_{1}, x_{3}\right), \lambda\right) \xi(\vec{x})=\left(\begin{array}{cc}x_{3}-x_{1}, & 0 \\ 0, & x_{3}-x_{2}\end{array}\right) \Gamma_{B}(\vec{x}, \lambda)\left(\begin{array}{cc}z_{3}-z_{1}, & 0 \\ 0, & z_{3}-z_{2}\end{array}\right)^{-1}$, where

$$
\begin{gathered}
\zeta(\vec{x})=(-1)^{\mu_{2}} \frac{\Gamma_{p}\left(1+x_{3}-x_{2}\right)}{\Gamma_{p}\left(1-x_{2}\right) \Gamma_{p}\left(x_{1}\right) \Gamma_{p}\left(x_{3}-x_{1}\right)} \cdot J_{1}(\vec{x}) J_{2}(\vec{x}), \\
J_{2}(\vec{x})=\left\{\begin{array}{ll}
p, & \mu_{3}<1+\mu_{2}, \\
\frac{1}{z_{3}-z_{2}}, & 1+\mu_{2} \leqslant \mu_{3},
\end{array} J_{1}(\vec{x})= \begin{cases}\frac{1}{p\left(z_{3}-z_{1}\right)}, & \mu_{3}<\mu_{1}, \\
1, & \mu_{1} \leqslant \mu_{3} .\end{cases} \right.
\end{gathered}
$$

Proof. This follows directly from Theorem 5 and [4, equation (1.11)].

7. The Kummer group. Let us refer to the group of mappings $(a, b, c) \mapsto$ $(\hat{\hat{a}}, \hat{\hat{b}}, \hat{\hat{c}}) \bmod \mathbf{Z}$ in Table I as the Kummer group. Let us refer to the group of 48 elements generated by these together with the twist $(a, b, c) \mapsto(b, a, c)$, as the extended Kummer group. We give an intrinsic distinction between these two groups (viewed as subgroups of $\mathrm{GL}(3, \mathbf{Z})$ ).

Let us view $0, a, b, c$ elements of $\mathbf{R} / \mathbf{Z}$, i.e. as points on the real circle. If $(a, b, c) \mapsto(\hat{a}, \hat{b}, \hat{c})$ denotes an element of the extended Kummer group then:

7.1. The points $0, c$ are separated by the points $a, b$ (on the circle) if and only if the points $0, \hat{c}$ are separated by the points $\hat{a}, \hat{b}$.

7.2. If the points $0, c$ are separated by the points $a, b$ then the orientation of $0, a, c, b$ coincides with that of $0, \hat{a}, \hat{c}, \hat{b}$ if $(a, b, c) \mapsto(\hat{a}, \hat{b}, c)$ lies in the Kummer group but not if it lies in the other coset of the Kummer group in the extended group.

7.3. RemarK. If the points $0, c$ are not separated by $a, b$ then the orientation of $\hat{a} \hat{b} \hat{c}$ and $a b c$ need not coincide for the Kummer group.

7.4. We may check properties 7.1 (resp. 7.2) for the extended (resp. nonextended) Kummer group by checking the generators $M_{13}, M_{7}$ of the Kummer group and the twist for the extended Kummer group.

A second verification of 7.1 for the extended Kummer group comes from the condition for the existence of two solutions mod $p$ of the hypergeometric differential equation. 
A third verification of 7.1 and 7.2 involves the dependence of the magnitude of $\operatorname{det} \gamma(\vec{a}, \vec{b}, \lambda)\left(\lambda\right.$ satisfying (1.9.1)) upon the relative magnitudes of $\mu_{1}, \mu_{2}, \mu_{3}$ (the representatives in $[0, p-1]$ of $\left.-a_{1},-a_{2},-a_{3}\right)$.

7.5. LEMMA. The extended Kummer group as a subgroup of $\mathrm{GL}(3, \mathbf{Z})$ is characterized by property 7.1. The Kummer group is characterized by the further property 7.2.

Proof. It is enough to show that there are at most 48 elements of $\operatorname{GL}(3, \mathbf{Z})$

$$
(a, b, c) \mapsto(\hat{a}, \hat{b}, \hat{c})=(a, b, c)\left(\begin{array}{lll}
l_{1} & m_{1} & n_{1} \\
l_{2} & m_{2} & n_{2} \\
l_{3} & m_{3} & n_{3}
\end{array}\right)
$$

which satisfy 7.1 .

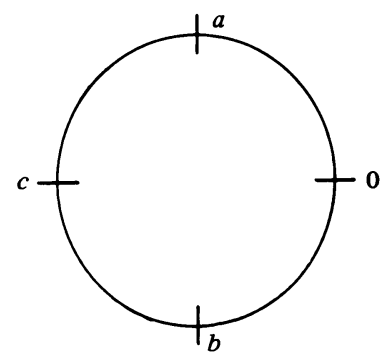

Consider $a, b$ separated by $0, c$ as indicated. Then $\hat{a}, \hat{b}$ (as given by (7.5.1) separate $0, \hat{c}$. Let $c, b$ be fixed and permit $a$ to approach 0 . When $a$ passes 0 , the points $\hat{a}, \hat{b}$ can no longer separate $0, \hat{c}$. Hence when $a=0$ the diagram $\hat{a}, \hat{b}, \hat{c}, 0$ must collapse. We assume here that $b$ and $c$ are generic. The collapse can occur in four ways,

$$
\begin{array}{ll}
\text { (1) } \hat{a}=0, \quad \text { i.e. } & b l_{2}+c l_{3}=0, \\
\text { (2) } \hat{a}=\hat{c}, & b l_{2}+c l_{3}=b n_{2}+c n_{3}, \\
\text { (3) } \hat{b}=0, & b m_{2}+c m_{3}=0, \\
\text { (4) } \hat{b}=\hat{c}, & b m_{2}+c m_{3}=b n_{2}+c n_{3} .
\end{array}
$$

Now clearly (1) and (2) cannot occur simultaneously and neither can (3) and (4). We assert that indeed no two can occur simultaneously. If (1) and (3) occur when $a=0$ then (since $b, c$ are generic) $l_{2}=l_{3}=0, m_{2}=m_{3}=0$ which shows that the transformation is not invertible, a contradiction. A similar analysis rules out the remaining $\left(\begin{array}{l}4 \\ 2\end{array}\right)-3$ pairs. In this way we deduce a mapping depending upon (7.5.1) of the set of 4 situations

$$
\begin{aligned}
& \text { (1) } a=0, \\
& \text { (2) } a=c, \\
& \text { (3) } b=0, \\
& \text { (4) } b=c
\end{aligned}
$$

into the set of 4 situations (7.5.2) in terms of $\hat{a}, \hat{b}, \hat{c}$. By using the inverse of (7.5.1) we conclude that there exists a mapping of the situations (7.5.2) into the situations 
(7.5.3), i.e. the mapping (7.5.1) satisfying 7.1 determines a one-to-one mapping of the situations (7.5.2) onto the situations (7.5.3). There are exactly $4 \cdot 3 \cdot 2 \cdot 1=24$ such mappings. On the other hand, a one-to-one correspondence between (7.5.2) and (7.5.3) determines the matrix of (7.5.1) up to a scalar factor. For example, suppose

$$
\begin{aligned}
& a=0 \Rightarrow \hat{a}=0, \\
& a=c \Rightarrow \hat{a}=\hat{c}, \\
& b=0 \Rightarrow \hat{b}=0, \\
& b=c \Rightarrow \hat{b}=\hat{c},
\end{aligned}
$$

then

$$
\begin{aligned}
l_{2} & =l_{3}=0, \quad l_{2}=n_{2}, \quad l_{1}+l_{3}=n_{1}+n_{3}, \\
m_{1} & =m_{3}=0, \quad m_{1}=n_{1}, \quad m_{2}+m_{3}=n_{2}+n_{3},
\end{aligned}
$$

from which we deduce that the matrix of (7.5.1) is a scalar, $l_{1}=m_{2}=n_{3}$.

Since the matrix (7.5.1) has determinant \pm 1 , there are at most two choices of (7.5.1) for each 1-1 correspondence between (7.5.2) and (7.5.3). This completes the proof.

\section{REFERENCES}

1. A. Adolphson and S. Sperber, Twisted Kloosterman sums and p-adic Bessel functions, Amer. J. Math. (to appear).

2. W. Burnside, Theory of groups of finite order, Dover, New York, 1955.

3. B. Dwork, Lectures on p-adic differential equations, Springer-Verlag, Berlin and New York, 1982.

4. __ On the Boyarsky principle, Amer. J. Math. 105 (1983), 115-156.

5. A. Erdelyi, Integral darstellungen hypergeometrischen functionen, Quart. J. Math. Oxford Ser. 8 (1937), 267-277.

6. Transformation of hypergeometric integrals by means of fractional integration by parts, Quart. J. Math. Oxford Ser. 10 (1939), 176-189.

7. A. Erdelyi, W. Magnus and F. G. Tricomi, Higher transcendental functions, Vol. I, McGraw-Hill, New York, 1953.

8. P. Monsky and G. Washnitzer, Formal cohomology. I, Ann. of Math. (2) 88 (1968), 181-217.

9. P. Monsky, Formal cohomology. II, Ann. of Math. (2) 88 (1968), 218-238.

10. Formal cohomology. III, Ann. of Math. (2) 93 (1971), 315-343.

11. E. Poole, Introduction to the theory of linear differential equations, Oxford Univ. Press, Oxford, 1936.

12. B. Riemann, Gesammelte Math Werke, Dover, New York, 1953.

Department of Mathematics, Princeton University, Princeton, New Jersey 08544 